\title{
„Die neue Angst vorm schwarzen Mann"
}

\author{
Moralpaniken als Reaktion auf Geflüchtete im Regierungsbezirk Tübingen[1]
}

\author{
Nikolai Huke
}

\begin{abstract}
Moralpanik bezeichnet eine Dynamik, im Zuge derer eine Gruppe als homogen, ,fremd', ,deviant' und Gefahr für die moralische Ordnung der Gesellschaft konstruiert wird. Der Artikel arbeitet am Beispiel von politischen Deutungskämpfen um Kriminalität und die Landeserstaufnahmeeinrichtung (LEA) Sigmaringen sowie um Vergewaltigungen in Tübingen typische Elemente der Moralpaniken im Bereich Flucht und Migration heraus: lange Äquivalenzketten, ein Generalverdacht gegenüber bestimmten Gruppen, raunende Sprache, die retrospektive Konstruktion einer vom Verfall bedrohten Idylle, der Rekurs auf einen vermeintlichen gesunden Menschenverstand, dem eine naive politische Korrektheit gegenübergestellt wird sowie die Behauptung eines permissiven und hilflosen Agierens der Justiz- und Sicherheitsbehörden. Soziale Medien wie Facebook, so wird gezeigt, tragen insbesondere durch (teils strategische) Interventionen rassistisch auftretender Accounts zu einer raschen Ausweitung von Moralpaniken bei.
\end{abstract}

Ersteinreichung: 4. September 2018; Veröffentlichung online: 15. Mai 2019

An English abstract can be found at the end of the document.

\section{Einleitung}

„Die neue Angst vorm schwarzen Mann“ übertitelt die Stuttgarter Zeitung einen Artikel zu Reaktionen auf Geflüchtete in Sigmaringen (Stuttgarter Zeitung 13.9.2016). Der Titel ist exemplarisch für ein Phänomen, das in der sozialwissenschaftlichen Kriminalitätsforschung als „Moralpanik“ bekannt ist: Eine Gruppe wird als homogen, ,fremd' und ,bedrohlich' konstruiert. Für Delikte werden nicht mehr einzelne Täter verantwortlich gemacht, sondern ein - im Prozess der Moralpanik konstruiertes - Kollektiv. Moralpaniken sind vor dem Hintergrund gesellschaftlich weit verbreiteter rassistischer Einstellungsmuster ein strukturierendes Element von Debatten über Flucht und Migration. Exemplarisch steht hierfür das diskursive Ereignis ,Silvester in Köln' (vgl. Arendt/Brosius/Hauck 2017). Am Beispiel von politischen Deutungskämpfen um Kriminalität und die Landeserstaufnahmeeinrichtung (LEA) Sigmaringen sowie um Vergewaltigungen in Tübingen untersucht der vorliegende Text die Entstehungsweise, Dynamik und Auswirkungen von Moralpaniken. Empirische Grundlage ist eine qualitative Analyse der Lokalpresse sowie von Facebook-Debatten auf der Seite des Tübinger Oberbürgermeisters Boris Palmer. 


\section{Diskursanalysen über Flucht und Migration in Deutschland}

Der Diskurs um Flucht und Migration in Deutschland wurde in den vergangenen Jahrzehnten in vielfältiger Weise zum Gegenstand sozialwissenschaftlicher Auseinandersetzungen. Schematisch lassen sich mindestens vier Zugriffe unterscheiden. Erstens liegen zahlreiche Arbeiten vor, die Topoi, Narrative und diskursive Ereignisse in der (vor allem print-)medialen Berichterstattung über Flucht und Migration in den Blick nehmen. Typische Narrative des flüchtlingspolitischen Diskurses der vergangenen Jahre (und teilweise der vorangegangenen Jahrzehnte) sind Natur- und Katastrophenmetaphorik, kulturelle Fremdheit (vor allem mit Bezug auf den Islam bzw. Muslime), Gewalt-, Kriminalitäts- und Terrorängste, Überlastung und Chaos, Asylmissbrauch, aber auch wirtschaftliche Nützlichkeit, Hilfsbereitschaft und Gastfreundschaft, Rassismus, Rechtsextremismus und ,besorgte Bürger' (vgl. exemplarisch die Beiträge in Jäger/Wamper 2017). Die unterschiedlichen Narrative sind mit Zuordnungen verbunden, wer für Leiden verantwortlich gemacht, wer Rechte und Repräsentation in Anspruch nehmen kann oder wer dazugehört und wer nicht (vgl. Homes/ Castañeda 2016: 13). Zweitens untersuchen zahlreiche Arbeiten antimuslimischen Rassismus in der medialen Berichterstattung über Flucht und Migration, wobei sie postkoloniale Theorien (z. B. Edward Saids Konzept des Orientalismus) mit feministischen Analysen verknüpfen. Traditionen kolonialen Rassismus, so eine grundlegende These dieser Arbeiten, setzen sich bis heute fort und finden sich in Bildern vergeschlechtlichter und rassifizierter ,Anderer' wieder. Am Beispiel der medialen Verhandlungen der Ereignisse auf der Kölner Domplatte in der Silvesternacht 2015/2016 verweisen entsprechende Arbeiten auf eine Verschränkung von Sicherheits-, Flucht- und Geschlechterdiskursen mit Rassismus in der Figur des ,gewalttätigen und sexistischen muslimischen Flüchtlings' (vgl. exemplarisch Dietze 2016, Mühe 2017). Drittens liegen qualitative und quantitative Studien zu Sprechpositionen im flüchtlingspolitischen Diskurs vor, die aufzeigen, dass der Diskurs von Männern dominiert wird, sicherheitspolitische Apparate (z. B. die Polizei) eine wichtige Rolle spielen, die AfD und ihre Themen teilweise deutlich überrepräsentiert sind und dass Geflüchtete selbst kaum zu Wort kommen (vgl. exemplarisch Drüeke 2016, Goebel 2018: 192, Haller 2017: 93-95[2]). Viertens wird in Analysen neonazistischer und autoritär-populistischer Diskursstrategien beobachtet, dass sich eine von gesamtgesellschaftlichen politischen Aushandlungsprozessen relativ entkoppelte Teilöffentlichkeit herausbildet, in der rassistischen und flüchtlingsfeindlichen Diskursen eine Leitfunktion zukommt (vgl. Glorius/Schondelmayer/ Dörfel 2018, Huke 2018, Salzborn 2017).

\section{Das Konzept der Moralpanik}

Die Debattenstränge zum Diskurs um Flucht und Migration in Deutschland überschneiden sich mit Thematiken des unter anderem von Stanley Cohen (1980) und Stuart Hall et al. (2002) geprägten Konzepts der Moralpanik. Dennoch liegen bisher nur vereinzelt Studien vor, die explizit auf Cohen und/oder Hall zurückgreifen (z. B. Perthus/Belina 2017). Anders als in der 
Analyse von Topoi und Narrativen sowie von antimuslimischem Rassismus in der medialen Berichterstattung stehen bei der Analyse von Moralpaniken weniger (historisch relativ stabile) Muster der diskursiven Formationen und der Ordnung des Diskurses des migrationspolitischen Feldes (z. B. in kolonialer Tradition stehende rassistische Konstruktionen der ,Anderen') im Vordergrund, sondern Brüche, Konstruktionsprozesse von Devianz und der alltägliche Kontext von Äußerungen. Gegenüber der quantitativen Analyse von Sprecherpositionen oder dem Fokus auf Teilöffentlichkeiten, in denen Aushandlungsprozessen eine untergeordnete Rolle zukommt, fokussieren Studien von Moralpaniken das Zusammenspiel von (mehr oder weniger strategisch handelnden) Akteuren und medialer Berichterstattung. Das Konzept der Moralpanik ermöglicht dadurch ein akteurszentriertes und prozessorientiertes Verständnis von Diskursverläufen.

Moralpanik bezeichnet einen Prozess, in dem ein mit Kriminalität verknüpftes Ereignis zum Symbol eines breiteren Prozesses des Verfalls, der sozialen Desintegration und der Zerstörung des gesellschaftlichen Zusammenhalts wird (vgl. Fitzgerald/Smoczynski 2015: 383). Als diskursives Schlüsselereignis prägt es die öffentliche Debatte in der folgenden Zeit, medial werden ähnliche Ereignisse thematisiert und hervorgehoben (vgl. Arendt/ Brosius/Hauck 2017). Als Kontrast zum beschriebenen Verfallsprozess dienen nostalgische Narrative einer durch soziale Stabilität und moralische Disziplin geprägten glorreichen Vergangenheit (vgl. McRobbie/ Thornton 1995: 561-563). Als Verursacher der Verfalls- und Desintegrationsprozesse wird eine in stilisierter und stereotyper Form präsentierte Gruppe identifiziert und als fremd, abweichend und gefährlich gekennzeichnet (z. B. eine rassifizierende Konstruktion von ,Muslimen', ,Arabern', ,Schwarzen“ oder ,Gambiern'). Die stereotype Darstellung essentialisiert, naturalisiert, fixiert ,Differenz' und etabliert symbolische Grenzziehungen der (Nicht-) Zugehörigkeit (vgl. Hall 2004). Differenzen der als Gruppe zusammengefassten Menschen (z. B. aufgrund von Klasse, (Sub-)Kultur, Bildung, Alter oder Geschlecht), die sich jeweils kontextspezifisch artikulieren, werden ausgeblendet und negiert (vgl. Hark/Villa 2017). Die Gruppen existieren somit nicht vordiskursiv, sondern werden erst im Zuge der Moralpanik als Gruppe konstituiert. Verschieden gelagerte Unsicherheiten und diffuse Ängste werden auf die konstruierte Gruppe projiziert (vgl. Perthus/ Belina 2017: 256). Moralpaniken legitimieren und ermöglichen darüber repressive Maßnahmen, stabilisieren gesellschaftliche Hierarchien und soziale Exklusion. Mit der Konstruktion der Fremdgruppe wird implizit oder explizit auch eine Wir-Gruppe konstituiert (vgl. Garland 2008: 16, McRobbie/ Thornton 1995: 571).

Moralpaniken können nicht-intendiert im Zuge alltäglicher Konflikte entstehen. In TV- und Printmedien, aber auch in politischen Aushandlungsprozessen, werden sie jedoch auch strategisch genutzt, um Aufmerksamkeit und Zustimmung zu sichern. Regierungsvertreter_innen relativieren ihre eigene Verantwortung für gesellschaftliche Probleme, indem sie marginalisierte Gruppen als Verursacher identifizieren (vgl. McRobbie/Thornton 1995). Kommerzielle Medien nutzen Moralpaniken, um Einschaltquoten und Verkaufszahlen zu steigern (vgl. Garland 2008: 15). Der Polizei kommt eine wichtige Rolle zu, da sie über Deutungsmacht bei der Kennzeichnung 
und Einordnung von Kriminalität und der Konstruktion krimineller Gruppen verfügt (vgl. Cohen 1980: 91). Polizeiliche Kriminalstatistiken werden in der Moralpanik zu einer zentralen Ressource im Ringen um diskursive Hegemonie und zu Belegen für oder gegen einen gesellschaftlichen Verfallsprozess (vgl. Hall et al. 2002). Für autoritär-populistische Akteure sind Moralpaniken die zentrale Strategie der Machtgewinnung und des Machterhalts. Helmut Dubiel bezeichnet ihre Strategie treffend als „umgekehrte Psychoanalyse“: „Die neurotischen Ängste, die kognitiven Verunsicherungen und Regressionsneigungen werden aufgegriffen und mit dem Zweck systematisch verstärkt, den Patienten nicht mündig werden zu lassen.“ (Dubiel 1986: 42)

Die Voraussetzung dafür, dass Sprechakte zu einer Moralpanik beitragen, ist, dass diffuse gesellschaftliche Sorgen und Ängste oder auch manifeste rassistische Deutungsmuster vorhanden sind, an die sie anknüpfen können (vgl. Fitzgerald/Smoczynski 2015). Kennzeichen entsprechender Sprechakte ist eine emotionalisierende Sprachverwendung (vgl. McRobbie/ Thornton 1995: 562). Begünstigende Faktoren sind sensationsorientierte Massenmedien, neue oder zuvor noch nicht medial präsente Formen von Devianz, marginalisierte Outsider sowie eine bereits für das entsprechende Thema sensibilisierte Öffentlichkeit (vgl. Garland 2008: 14). Die Fremdgruppenkonstruktion im Zuge der Moralpanik erfolgt in der Regel nicht ad hoc, sondern greift auf ein Archiv aus tradierten (z. B. (kolonial-)rassistischen) Diskursen zurück. Daher erweist es sich als produktiv, die Analyse von Moralpaniken mit den von diskursanalytischen Studien herausgearbeiteten Narrativen im flüchtlingspolitischen Diskurs zu verknüpfen, die in der alltäglichen Deutung von Ereignissen ein etabliertes Repertoire an Assoziationsketten zur Verfügung stellen. Gesellschaftlich weit verbreitete antimuslimische Ressentiments ermöglichen es etwa, Ereignisse durch die schon vorhandene Wissensordnung des antimuslimischen Rassismus zu filtern (vgl. Dietze 2016: 96).

Soziale Medien haben den Ablauf von Moralpaniken verändert (vgl. Cohen 2011: 241). Ihre Kommunikationsräume sind im Gegensatz zu TV- und Printmedien - was zunächst kontraintuitiv erscheint - teils weniger pluralistisch und dialogisch angelegt: Einerseits sind die Nutzer_innen selbst wesentlich mit ähnlich eingestellten Nutzer_innen verknüpft (Echokammern), andererseits bevorzugen die Algorithmen der Seiten Inhalte, die für die Nutzer_innen aufgrund seines Nutzungsverhaltens von Interesse sein dürften (Filterblasen) (vgl. Amadeu Antonio Stiftung 2017: 9). Eher als zu Diskussion und Austausch dienen soziale Medien in der Folge dazu, Emotionen und Symbole zu mobilisieren und zu verbreiten (vgl. Mühe 2017: 32). Die Feedbackschleifen der sozialen Medien verleiten in Deutschland völkisch-konservative und neonazistisch eingestellte Personengruppen zu immer radikaleren Ausfällen, wobei nicht selten versucht wird, Moralpaniken zu inszenieren (vgl. Salzborn 2017: 197). Beiträge werden dabei potenziell massenhaft geteilt, ohne dass Quellen geprüft werden (Gefahr von Fake News) (vgl. Salzborn 2017: 121). Während Moralpaniken traditionell durch Eliten diskursiv vorangetrieben wurden, können sie in sozialen Medien auch gegen das (vermeintliche) Verschweigen von Ereignissen durch Eliten gerichtet sein (vgl. Cohen 2011: 242). 
Moralpaniken weisen häufig eine räumliche Komponente auf. So werden etwa in der Studie von Cohen (1980) britische Badeorte durch die Problematisierung von Mods und Rockern medial zu Gefahrengebieten. Hall et al. (2002) beschreiben, wie einzelne Stadtviertel in der Diskussion um Raubüberfälle (mugging) zu Problembezirken erklärt werden. Moralpaniken fungieren dadurch als Antriebskraft kontrollpolitischer Raumstrategien, die gegenüber problematisierten Gruppen auf Verbannung, polizeiliche Regulierung und Stigmatisierung setzen. Die aus Ausgrenzungsstrategien resultierende räumliche Trennung geht mit Erfahrungsbarrieren einher, die die Entstehung von Bedrohungsszenarien (z. B. sogenannte AusländerGhettos) vereinfachen (vgl. Tsianos 2013: 27). In ihrer medialen Verhandlung werden Orte gleichzeitig als eingegrenzte Räume hervorgebracht und transzendiert. Moralpaniken um einzelne Orte (z. B. ,Silvester in Köln') „reisen“ (Butler 2011, Übers. d. A.) - insbesondere in digitalen sozialen Medien - und bedingen anderswo eine Re-Konfiguration oder emotionale Neubewertung von Raumordnungen (z. B. Konstruktion von ,muslimischen Flüchtlingen' als Problem in Schwimmbädern oder Clubs und anschließende Ausschlussmechanismen).

\section{Fallstudien}

Im Zuge des ,Sommers der Migration“ kam es 2015 im Regierungsbezirk Tübingen zu einem starken Zuzug von Geflüchteten. An politischen Auseinandersetzungen um Kriminalität und Geflüchtete, die in diesem Zusammenhang auftraten, lassen sich exemplarisch Verlauf und Konsequenzen von Moralpaniken beobachten. Eine zentrale Position in den Deutungskämpfen kommt dem Tübinger Oberbürgermeister Boris Palmer zu, dessen Äußerungen in Printmedien und auf Facebook zu Referenzpunkten werden, an denen um Moralpaniken gerungen wird.

Im Folgenden werden zwei Beispiele vorgestellt: Die Debatte um Kriminalität und die LEA in Sigmaringen sowie die Diskussion um Vergewaltigungen durch einen aus Gambia stammenden Mehrfachtäter in Tübingen. Die Beispiele wurden im Rahmen einer umfassenderen Analyse erarbeitet. Zunächst wurden über eine qualitative Auswertung von Zeitungsartikeln eines über die Datenbank LexisNexis generierten Samples zentrale diskursive Ereignisse des Flüchtlingsdiskurses im Regierungsbezirk Tübingen identifiziert. Von diesen wurden vier für eine Feinanalyse ausgewählt, in denen Kriminalität und/oder Gewalt eine zentrale Rolle spielten (neben den beiden vorgestellten Ereignissen noch die Ereignisse,Dönermesser-Mord' in Reutlingen und sexualisierte Übergriffe im Epplehaus). Zu den Ereignissen wurden jeweils die Berichterstattung der auflagenstärksten Lokalzeitung sowie ein thematisch einschlägiger Eintrag auf der Facebookseite Boris Palmers inklusive aller Kommentare über eine offene Codierung in MAXQDA ausgewertet. Während die analysierten Printmedien (z. B. durch die Verbreitung ihrer Printausgabe) lokal beschränkt sind, sind die Debatten auf Facebook durch die Reichweite der Seite Boris Palmers Teil bundesweiter Gesamtdiskurse. Der Gegenstand weist damit über den Regierungsbezirk Tübingen hinaus. Printmedien und Facebook sind analytisch nur begrenzt trennbar: Einerseits kommt Printmedien eine Leitfunktion in sozialen 
Medien zu, andererseits übernehmen die untersuchten Printmedien wiederholt Zitate aus Facebook-Einträgen von Boris Palmer.

Die Analyse wurde aus forschungspragmatischen Gründen diskursanalytisch beschränkt: Weder das Wechselverhältnis der beschriebenen Moralpaniken zu alltäglichen Praktiken im städtischen Raum im Regierungsbezirk Tübingen, noch eine systematische Ver-Objektivierung der beschriebenen Ereignisse über eine Ereignisbeschreibung, die über dessen mediale Repräsentation hinausweist, konnten an dieser Stelle geleistet werden. Eine Trennung von Kontextinformationen, inhaltlich-deskriptiver Analyse sowie der Analyse von Diskursstrategien und -mustern erweist sich bei der Analyse von Moralpaniken grundsätzlich als schwierig, da statistisches Material vom Anzeigeverhalten der Bevölkerung und polizeilichen Kontrollstrategien abhängt, die beide potentiell stark mit einer im Zuge der Moralpanik generierten Aufmerksamkeit für eine bestimmte Problematik korrelieren (vgl. Hall et al. 2002: 9-10). In der Darstellung der beiden Ereignisse werden zunächst eher beschreibende Diskurselemente vorgestellt, anschließend wird der Fokus auf die Analyse von Diskursstrategien und -mustern gelegt. Das sollte jedoch nicht darüber hinwegtäuschen, dass Beschreibung und (vermeintlich objektive) Statistiken Teil und Effekt diskursiver Aushandlungsprozesse sind.

\subsection{Kriminalität in Sigmaringen}

2016 steigt nach Angaben der Polizei die Anzahl der Straftaten pro 100.000 Einwohner im Landkreis Sigmaringen von 3977 auf 4444, bleibt damit aber immer noch unter dem Landesdurchschnitt (5390) (vgl. Schwäbische Zeitung 18.3.2017). Für die Stadt Sigmaringen steigt sie auf 9825 (vgl. Schwäbische Zeitung 23.3.2017). Die Anzahl der tatverdächtigen Asylbewerber_innen steigt im gleichen Jahr im Bereich des Polizeipräsidiums Konstanz deutlich an, von insgesamt 19203 Tatverdächtigen[3] waren 1943 Asylbewerber_innen. Besonders ausgeprägt ist die Zunahme in Sigmaringen. Ein Großteil der Straftaten - in der Regel Diebstahldelikte und Körperverletzungen - wird innerhalb von Flüchtlingseinrichtungen begangen (vgl. Schwäbische Zeitung 18.3.2017). Rund um die LEA Sigmaringen beobachtet die Polizei im Februar 2017 eine Zunahme von Delikten („Diebstähle, Körperverletzungen, Saufereien“, Schwäbische Zeitung 17.2.2017). Von Anfang Januar 2017 bis Mitte Februar kommt es zu 100 Polizeieinsätzen in der LEA (vgl. ebd.). Eine eigene Ermittlungsgruppe mit zehn Beamt_innen kümmert sich um Straftaten in und um die LEA (vgl. ebd.). 2017 steigt die Kriminalität in der Stadt Sigmaringen im Vergleich zum Vorjahr um knapp 36 Prozent, den stärksten Anstieg verzeichnen Rauschgiftdelikte (plus 70 Prozent). Rund 57 Prozent der Tatverdächtigen in Sigmaringen sind Flüchtlinge, im Land liegt diese Zahl bei etwa zehn Prozent (vgl. Schwäbische Zeitung 16.5.2018).

Die Schwäbische Zeitung berichtet ab Anfang 2017 verstärkt über Gewalt und Kriminalität durch Flüchtlinge der LEA Sigmaringen (z. B. „LEA: Drogen, Gewalt und Brandstiftung“, Schwäbische Zeitung 17.2.2017). Anfangs werden Probleme innerhalb der Unterkunft selbst thematisiert, in der Folge Kriminalität durch Flüchtlinge außerhalb der LEA. Ende 2017 geraten das Bahnhofsgebiet und der Prinzenpark in den Fokus, die Schwäbische Zeitung 
berichtet von „Rangeleien, Pöbeleien, Trinkgelage[n], Drogendelikte[n], Raubüberfälle[n], Körperverletzungen" (Schwäbische Zeitung 29.11.2017). Geflüchtete werden diskursiv zunächst vor allem im Zuge von polizeilichen Einsätzen und Ermittlungen sichtbar. In der Folge erscheinen sie als „,aufgebracht“ (Schwäbische Zeitung 17.1.2017) bzw. „,betrunken“ und „aggressiv“ (Schwäbische Zeitung 29.1.2017). Polizei, aber auch Richter finden hierfür vor allem zu Beginn der Auseinandersetzung kulturalisierende Erklärungsmuster. Der Polizeipräsident identifiziert „mangelnden Respekt vor meinem Gegenüber“ als Ursache (vgl. Schwäbische Zeitung 10.2.2017). Ein Verwaltungsrichter erklärt: „Die Flüchtlinge kommen in eine Welt, die sie so nicht gewohnt sind." Nach Angaben der Schwäbischen Zeitung meint er damit „auch den Stellenwert, den Frauen in Deutschland haben“(ebd.).

Im weiteren zeitlichen Verlauf der Debatte hebt die Polizei soziale Ursachen der Kriminalität hervor und betont, dass es sich um eine „kleine Gruppe“ handele, die verantwortlich sei: „Es gibt eine kleine Gruppe von marokkanischen Staatsangehörigen, die immer wieder auffällt [...]. Man geht davon aus, dass die Bleibeperspektive dieser jungen Männer eher düster aussieht und dass Frust eine Rolle spielt." (Schwäbische Zeitung 16.2.2017) Es gebe nicht „die straffälligen Flüchtlinge“, das Gros der Fälle gehe auf das Konto von 30 aus Marokko, Gambia und Eritrea stammenden Männern (vgl. Schwäbische Zeitung 23.3.2017). Kriminalität lasse sich jedoch „nicht anhand der ethnischen Herkunft bestimmen" (Schwäbische Zeitung 3.7.2017).

Der Leiter der LEA stellt soziale Ursachen in den Mittelpunkt. Die Zunahme von Straftaten ergebe sich daraus, dass am Anfang wesentlich mehr Familien gekommen seien. Diese seien stabiler aufgestellt und neigten weniger zu Eskapaden. Inzwischen gebe es mehr junge Männer (vgl. Schwäbische Zeitung 16.2.2017). Er bemängelt ein fehlendes Interesse der Anwohner, mit den Flüchtlingen selbst zu sprechen. Probleme bereite nur ein kleiner Teil der Flüchtlinge (vgl. Schwäbische Zeitung 6.4.2017). Die zuständige Mitarbeiterin für Presse- und Öffentlichkeitsarbeit in der Einrichtung weist verallgemeinernde Erklärungen zurück, es seien nicht ,die Marokkaner", die Probleme bereiten: „Ich denke, man muss schon das Individuum sehen“ (ebd.). Flüchtlingshelfer kritisieren ebenfalls eine unzulässige Pauschalisierung (z. B. „Alle Flüchtlinge werden in einen Topf geworfen“, Schwäbische Zeitung 17.2.2017).

In Berichten über Äußerungen von Anwohner_innen, Bahnkund_innen oder Gewerbetreibenden werden vor allem negative Auswirkungen der LEA hervorgehoben (z. B. „Man muss zuschauen, wie das gesamte Gebiet verkommt“, Schwäbische Zeitung 17.2.2017). Dabei wird eine zunehmende gefühlte Unsicherheit an bestimmten Orten, insbesondere dem Bahnhof, thematisiert (z. B. „Alkohol ist ein großes Problem [...]. Ein Mann wollte sich mit mir unterhalten und er hat nicht kapiert, dass ich dies nicht will“, Schwäbische Zeitung 29.11.2017). Insgesamt räumt die Berichterstattung der Schwäbischen Zeitung dem veränderten Sicherheitsgefühl von Anwohner_innen und Gewerbetreibenden relativ viel Raum ein:

„Ralf Fessler übte mit deutlicher Schärfe Kritik an den Zuständen. Die Nachtruhe bei seinem Haus in der Riedlinger Straße werde ständig von betrunkenen, schreienden Flüchtlingen gestört, seine Frau und 
Tochter seinen belästigt worden und hätten besonders abends Angst. Er wolle deshalb sein Haus verkaufen. [...] ,Die Leute verlassen die Stadt, der Ruf der Stadt ist am Boden', sagte Fessler." (Schwäbische Zeitung 10.1.2018)

Die Polizei wirkt gegenüber diesen Stimmen eher deeskalierend, die deutschen Gesetze würden auch für Flüchtlinge gelten („Wir müssen uns an geltendes Recht halten.“, vgl. Schwäbische Zeitung 10.1.2018). Darüber zeigt sie sich teilweise bemüht, das subjektive Unsicherheitsempfinden durch Verweise auf statistische Wahrscheinlichkeiten zu reduzieren (z. B. „Die Wahrscheinlichkeit, Opfer einer Straftat zu werden, ist aber wirklich gering. Man braucht keine Angst zu haben.", Schwäbische Zeitung 19.4.2018)

Sigmaringens Bürgermeister Thomas Schärer (CDU) erklärt, er sei erstaunt, dass es auf dem riesigen Kasernen-Areal nicht möglich sei, eine Zone für den Alkoholkonsum auszuweisen, wodurch „sich am Bahnhof ein entsprechendes Klientel [sic!] versammelt" (Schwäbische Zeitung 20.11.2017). Die Stadtverwaltung stellt fest, dass „Alkoholkonsum, damit einhergehende Hemmungslosigkeit, Missachtung des Rauchverbotes und Pöbeleien [...] dazu [führten], dass das Bahnhofgebäude von Reisenden nicht mehr seiner Zweckbestimmung entsprechend genutzt wird“ (Schwäbische Zeitung 8.2.2018). Schärer fordert neue Formen der Kontrolle. Notwendig seien verpflichtende Alkoholpräventionskurse, Benimmkurse, eine Verschärfung des Tatbestands der Beleidigung, Smartphone-Entzug und Hausarrest sowie die Einführung eines kommunalen Ordnungsdienstes (vgl. Schwäbische Zeitung 9.2.2018). Innenminister Thomas Strobl (CDU) und die Landtagsabgeordnete Andrea Bogner-Unden (Grüne) weisen die Vorschläge „aus verfassungsrechtlichen Gründen“ - beziehungsweise damit „die Grundrechte gewahrt bleiben“ - zurück (vgl. Schwäbische Zeitung 21.2.2018; Schwäbische Zeitung 4.3.2018).

Geflüchtete selbst kommen nur in einem Interview mit einem in der LEA untergebrachten Asylbewerber sowie einer Reportage über die Situation am Bahnhof zu Wort:

„Es gibt in der Kaserne praktisch keine Privatsphäre. [...] Wer sich nicht beschäftigen kann, entwickelt Aggressionen.“ (Schwäbische Zeitung 19.1.2017)

„Es ist ziemlich kalt an diesem März-Nachmittag, aber der Afrikaner am Bahnsteig macht keine Anstalten, ins Warme zu gehen. In der Hand hat der Mann mit den Rastalocken eine halbvolle Wodkaflasche [...]. Ein paar Minuten später steht er vor der Tür des Bahnhofs, während ein älteres Paar die Stufen zur Wartehalle hinaufsteigt. ,Hallo, alles gut?‘, ruft Bas ihnen zu. ,Halt dein Maul!', kommt es zurück. Drei bis vier Flaschen täglich trinke er [...]. Vor einem Jahr und zwei Monaten sei er nach Deutschland gekommen. Auf dem Weg von Afrika nach Italien sei sein Schiff gekentert. Hundert Menschen seien gestorben, 45 hätten überlebt. Seitdem trinke er. [...] Warum er am Bahnhof trinkt? Weil es in der Erstaufnahmestelle nicht erlaubt ist. ,Geht raus, sagen sie, geht in den Park oder sonst wohin. “ (Schwäbische Zeitung 29.3.2018) 
Die Debatte um Kriminalität und Geflüchtete der LEA Sigmaringen in der Schwäbischen Zeitung weist Elemente einer Moralpanik auf. Berichte über Stimmen aus der Bevölkerung werden von pauschalisierenden Zuspitzungen und gefühlter Unsicherheit dominiert (z. B. „Die Leute verlassen die Stadt, der Ruf der Stadt ist am Boden“), der Bürgermeister fordert extralegale Maßnahmen für Prävention und gegen ,kriminelle Flüchtlinge ‘ (z. B. „Smartphone-Entzug und Hausarrest“). Polizei, Mitarbeiter_innen der LEA sowie Flüchtlingshelfer_innen argumentieren demgegenüber differenzierend und betonen, dass es sich um eine kleine Problemgruppe handele, dass es soziale Ursachen für das Verhalten dieser Gruppe gäbe (z. B. „Bleibeperspektive [...] eher düster") und dass sich das Verhalten nicht pauschalisieren lasse (z. B. „Individuum sehen“). Es gibt eine für Moralpaniken charakteristische Verknappung der legitimen Sprecherpositionen und Sprechakte innerhalb des diskursiven Felds. Die Polizei dominiert die Berichterstattung, Positionen von Geflüchteten und Helfer_innen (z. B. „Hundert Menschen seien gestorben, 45 hätten überlebt. Seitdem trinke er.“) wird hingegen nur ein begrenzter Raum eingeräumt. Die Debatte ist deutlich männerdominiert, von 50 im Sample namentlich genannten Personen sind 37 männlich (74 Prozent) und 13 weiblich.

Boris Palmer greift die Debatte am 5. Mai 2017 bei Facebook auf.[4] Er postet ein Foto junger Männer mit folgendem Begleittext:

„Sigmaringen. Bahnhof. Fünf junge Männer. Offensiver Auftritt. Kontrolle im Zug: Keiner hat einen Fahrschein. Zugfahrten haben sich verändert in den letzten Jahren. Ist es rassistisch, das zu beschreiben? Ist es fremdenfeindlich, sich dabei unwohl zu fühlen?“

Zu einem späteren Zeitpunkt ergänzt er den Beitrag um folgenden Text:

„PS: Warum so ein Beitrag mit Bild? Ich glaube, dass sehr viele Menschen diese Veränderung spüren und negativ bewerten. Wenn man das aber zu erkennen gibt, passiert das, was man hier auf 200 Kommentaren nachlesen kann: Massive moralische Verunglimpfung. Ich halte das für falsch. Probleme löst man nicht durch Wegsehen. Und diese Problem sid[5] real. Warum ein Foto? Weil es die Szene verständlich machen soll. Hätte ich stattdessen geschrieben, dass es fünf junge Männer waren, die aussahen wie aus dem Maghreb, arabisch Sprachen, durch die Kontrolle als Asylbewerber erkennbar wurden - was wäre dann bitte los gewesen? Diese selbstgerechten Empörungsstürme lösen kein Problem. Sie schaffen neue. Und wer mit Leuten spricht, die das täglich erleben, der erfährt: Gerade angelehnte Asylbewerber dieser Gruppe halten sich wenig an Regeln. Sie wissen, dass Ihnen nichts passiert.“

In einem Folgebeitrag verknüpft er seine Darstellung explizit mit der LEA Sigmaringen:

„Hinzu kommt, dass gerade in Sigmaringen eine größere Gruppe von Asylbewerbern aus dem Maghreb für erheblich Probleme nicht nur in der Stadt sondern auch in der LEA gesorgt hat. Wenn man das weiß, fühlt man sich doppelt unwohl, wenn solche Gruppen am Bahnhof den öffentlichen Raum für sich einnehmen." (Palmer 6.5.2017) 
Der Beitrag erhält 2931 Reaktionen (,Likes', usw.) und wird 716-mal geteilt. Die Debatte unter dem Beitrag wird von Männern dominiert: Von 1903 Kommentaren wurden 1392 von männlichen Profilen verfasst (73,15 Prozent), davon 221 von Boris Palmer (11,61 Prozent). Demgegenüber standen 403 von weiblichen Profilen (21,18 Prozent) und 108 von Profilen, deren Namen nicht klar zugeordnet werden konnten (Stand: 13.3.2018).

Bezüglich der Debatte um Flucht und Asyl finden sich unter dem Beitrag Äquivalenzketten zwischen Fahren ohne Fahrschein, Kriminalität, Gewalt, ,Gangs' und schließlich Terroranschlägen. Boris Palmer selbst legt diese Interpretation in einigen seiner Kommentare nahe: „Schwarz fahren ist nur ein randaspekt. [...] Ich weiß, dass in Sigmaringen ein grosse Gruppe von Asylbewerbern aus dem Maghreb massiv für Ärger gesorgt hat. Auch in der LEA. Da entsteht ein Bedrohungsgefuhl“. Folgebeiträge konstruieren ergänzend ein Kontinuum, das von Fahren ohne Fahrschein bis Terrorismus reicht:

„Und dabei geht es nicht nur ums Schwarzfahren, das auch mich betrifft (als ehrlich zahlender Fahrgast muss ich diese Kosten im Fahrkartenpreis mittragen). Gerade die muslimischen geprägten Personen sind für Terroranschläge bereit (z. B. Paris, Nizza, Berlin, Würzburg usw.) was in keinster Weise hier zu uns nach Deutschland passt.“ (Stefan B.)

Zentral für die Debatte ist die Konstruktion, dass rechtsfreie Räume drohten. Nahe gelegt wird dies wiederum von Boris Palmer selbst: „Das schwarz fahren ist dann ein Symptom für: Unsere Regeln sind denen egal, der Staat findet darauf aber keine Antwort“. In den Kommentaren wird diese Konstruktion vielfach aufgegriffen und teils weiter zugespitzt, etwa folgendermaßen: „Es kommen hier Leute ins Land, die interessieren sich nicht für unsere Regeln (vom Grundgesetz brauchen wir erst gar nicht reden) und terrorisieren unser Leben“(Markus Z.) oder „Ich denke, es war rückblickend gesehen sehr falsch, dass die ersten Gerichtsurteile für abgelehnte Asylbewerber alle doch sehr milde ausgefallen sind“"(Petra N.). Die Konstruktion legitimiert Forderungen nach Abschiebungen:

„Warum hat man die Bevölkerung nicht gefragt, ob sie jährlich über 20 MILLIARDEN für sogenannte ,Flüchtlinge‘ ausgeben will? [...] Von den Milliarden Umsätzen der ASYLINDUSTRIE möchte ich erst gar nicht sprechen. Armes Deutschland. Falsche Toleranz gegenüber Intoleranten ist der Anfang vom Untergang einer Gesellschaft!!!!!!“ (Jürgen W.)

Mit der von rassistischen Deutungsmustern durchdrungenen Debatte um Flucht und Asyl werden gleichzeitig drei weitere Topoi verschränkt: Kulturelle Fremdheit, Angsträume sowie gesellschaftlicher Verfall. Alle drei Topoi werden von Boris Palmer selbst eingeführt. Kulturelle Fremdheit wird von ihm als Problem von Sprache und Einstellung gefasst (z. B. „Fangen an aufeinander zu schlagen, reden laut, alles auf Arabisch“, „Weil ich mich seltsam fühle, wenn Arabische die Sprache des Bahnhofs ist"). Der argumentative Faden wird in weiteren Beiträgen fortgeführt und über (vermeintliche) Augenzeugenberichte zugespitzt: 
„Vor kurzem fuhr ich seit langer Zeit, sonntags, in einem Regiozug. [...]. Fast nur arabische Menschen. [...] Der Lärmpegel war fast unerträglich. Schreiende und tobende Kinder, die auf den Sitzen rumsprangen und die Fensterscheiben verschmierten. Essensverpackungen und Essenreste wurden auf den Boden geschmissen. Sitze wurden mit Essen und Getränken beschmiert.“ (Ilke S.)

„Bedrohungsgefühl“ und „Unwohlsein im öffentlichen Raum“verdichten sich zum Narrativ von durch Zuwanderung entstandenen Angsträumen, die es zu meiden gelte:

„Das verhindert allerdings, ein WC in der Bahn zu nutzen. So entgehen Frauen Vegewaltigungsversuchen durch Migranten [...] wenn dann noch Axt- oder messerschwingende Islamisten u.a. auf die Fahrgäste losgehen, was sich mittlerweile auch häuft, stelle ich mir die Frage ,bin ich rassistisch'. Die Antwort ist: ,JA“.” (Meira E.)

Der Topos eines gesellschaftlichen Verfalls wird von Boris Palmer im Eingangspost eingeführt („Zugfahrten haben sich verändert“) und in seinen Kommentaren erneut aufgerufen („mein lebensumfeld verändert sich dadurch sehr negativ“). Die Begründung der Topoi Fremdheit, Angsträume und gesellschaftlicher Verfall erfolgt wesentlich auf der Grundlage einer anekdotischen Empirie, die selektiv mit Kriminalstatistiken verknüpft und darüber vermeintlich zu einer objektiven Tatsache wird. Die polizeiliche Kriminalstatistik wird in diesem Zusammenhang zu einer zentralen Legitimationsinstanz. Kritik an der eigenen Wahrnehmung wird abgewehrt, indem denjenigen, die die entsprechende Perspektive nicht teilen, Realitätsverweigerung unterstellt wird: „Polizei Statistik Sachsen. 60\% der Gruppe kriminell. [...] Und die Zahlen in NRW sind ähnlich. Jetzt recherchieren Sie mal“ (Boris Palmer). „Statistik“ fungiert dabei teilweise als abstrakte Begründung für die eigene gefühlte Wahrheit: „Irreale Ängste? [...] Schauen sie mal in die Statistiken umd sie sehen ganz reale Ängste“ (Benjamin Y.).

Durch die Konstruktion einer vermeintlichen „extremistischen politischen Korrekheit“ (Torsten M.), die eine sachliche Debatte verhindere, wird die eigene Wahrnehmung gegen Kritik immunisiert:

„Herr Boris Palmer spricht für viele Deutschen Bürger. Auch ich erleben es immer wieder, dass sich viele nicht trauen das Thema in der Öffentlichket und nur hinter vorgehaltener Hand under Bekannten, Freunden zu sprechen weil sie Angst haben als Arassit [Rassist] beschimpft zu werden." (Oliver W.)

Der Konstruktionsprozess einer realitätsfernen politischen Korrektheit impliziert eine klare Feindkonstruktion: die „Linksverwirrten und vielen Grünen“ (s.o.), „Merkelianer“ und „Idealisten“ sowie „,selbstgerechte Weltverbesserer, aus der 68er Zeit“ (Philipp R.), „Gutmenschen“ und „moralische Narzissten“ (Stefan B.), „Moralapostel“(Lukas P.) oder „Bahnhofsklatscher“(Andre O.). Dem imaginierten politischen Gegner wird zugeschrieben „Sprech- und Denkverbote [zu] bevorzugen“(Uwe S.), andere mit der „Nazikeule“ (Sebastian U.) zu verfolgen, „Multikulti-Gelaber“ (Stefan B.) zu praktizieren, „weltfremd“ (Axel M.) zu sein sowie mit „Tabu“ und „moralischer Überheblichkeit“ eine 
„verantwortungslose[...] gesinnungsethische[...] Einwanderungspolitik“ (Till H.) zu verfolgen. Rassistische und/oder autoritäre Positionen werden dadurch als Ausdruck des gesunden Menschenverstands in Szene gesetzt, die weder rechts noch rassistisch seien (z. B. „Sicherheitsgefühl ist subjektiv und daher nicht rassistisch!“; Peter M.). Die Bestätigung der eigenen Position durch Echokammern und Filterblasen legitimiert das Gefühl, Tabus gegenüberzustehen, die durchbrochen werden müssten:

„Wenn man einfach nur schildert, was man tAglich erleben kann, und dann sagt, da fühle ich mich unwohl, das ist ein unschöne Entwicklung, dann wird man bereits als rassist und fremdenhasser nieder gemacht. Man muss nur diesen Thread lesen, um zu sehen, worum es mir geht“ (Boris Palmer).

Die von Boris Palmer im Eingangsbeitrag aufgeworfene Frage „Ist es rassistisch, das zu beschreiben?" hat vor diesem Hintergrund eine doppelte Funktion: Einerseits delegitimiert sie all diejenigen Stimmen, die sie mit „Ja“ beantworten im oben ausgeführten Sinne als politisch korrekt, andererseits erklärt sie die eigene Position („Nein“) als nicht-rassistisch bzw. gerechtfertigt: „Absolut nicht rassistisch...sondern notwendig, Dinge beim Namen zu nennen!“ (Rainer N.); „dieses Verhalten hat mit dieser Gruppe zu tun, auch wenn es andere gibt. Das ist nicht rassistisch, sondern Realität“ (Boris Palmer) oder „Wenn Leute in unser Land kommen und unsere Regeln missachten, ist es kein Rassismus, dies zu kritisieren“ (Markus H.). Rassismus wird dadurch zu einer legitimen subjektiven „Meinung“, wer ihn benennt „denunziert“ (Boris Palmer): „Wo bleibt die Toleranz der Grünen? Andere Meinung : Rassist“ (Helmut K.).

Die Debatte um den Eintrag von Boris Palmer verstärkt die Moralpanik: Erstens wird das konkrete benannte Problem („Schwarzfahren“) emotional aufgeladen. Fahren ohne Fahrschein, eigentlich ein „Nicht-Ereignis“ (Cohen 1980: 9, Übers. d. A.), wird durch einen Prozess der „freien Assoziation“ (ebd.: 53) zu einem Symbol einer umfassenderen gesellschaftlichen (Negativ-)Entwicklung, eines ganzen „Spektrums an Problemen und Abweichungen“ (ebd.: 54). Nach Stanley Cohen sind entsprechende Dynamiken des „It's not only that" zentrales Element von Moralpaniken (vgl. ebd.: 53). In ihnen werden in einem diskursiven Selektionsprozess einem bestehenden Repertoire neue Elemente hinzugefügt und bestehende Narrative ausgeweitet (vgl. ebd.: 39). Zuvor als unauffällig gewertete Erfahrungen und Ereignisse werden reinterpretiert und als deviant bzw. gefährlich gekennzeichnet (vgl. ebd.). Der Beitrag von Boris Palmer eröffnet dadurch ein Sagbarkeitsfeld für - teilweise kulturalisierende und/oder rassistische - Konstruktionen von Fremdheit, von Angsträumen und von gesellschaftlichem Verfall. Migration wird zu einem bedrohlichen Phänomen (z. B. wenn das „Arabische die Sprache des Bahnhofs ist“). Die Erzählung Palmers wird dabei zweitens Teil von Äquivalenzketten, die Flucht bzw. Migration mit Fahren ohne Fahrschein, Kriminalität, Gewalt, Gangs und schließlich Terroranschlägen verknüpfen. Drittens werden in der Debatte subjektive Erfahrungen mit Statistiken zu gefühlten Wahrheiten verklammert. Dabei werden subjektive Narrationen (z. B. „Lärmpegel unerträglich“, „reden laut auf Arabisch“) über - teils real-existierende, teils gefühlte - Statistiken, 
insbesondere die polizeiliche Kriminalstatistik, legitimiert.[6] Viertens werden die entsprechend konstruierten ,Wahrheiten' als diskursiv nicht verhandelbare, einzig legitime Position inszeniert, indem jedwede Kritik als realitätsfremder Moralismus eines (unsachlich argumentierenden) politischen Gegners zurückgewiesen wird („political correctness“). Die selbsterklärte Intention Boris Palmers, durch seinen Beitrag zu einer offenen Auseinandersetzung über gesellschaftliche Probleme mit Asylbewerbern in Sigmaringen beizutragen, wird dadurch füftens in ihr Gegenteil verkehrt. Statt eine sachliche Debatte zu eröffnen, wird der Beitrag Teil eines antiaufklärerischen Narrativs, in dem nur noch jene Aspekte der Realität als wahr gelten, die die eigene Meinung stützen. Sechstens wird ein vermeintlich permissives und hilfloses Agieren der Justiz- und Sicherheitsbehörden kritisiert, womit implizit die Forderung nach einem Ausbau repressiver Staatlichkeit verbunden ist (vgl. Hall et al. 2002).

\subsection{Vergewaltigungen in Tübingen}

Im Juli 2017 wird in Tübingen ein Asylbewerber aus Gambia gefasst, der mehrerer Vergewaltigungen zwischen Mai 2015 und Februar 2017 überführt wird. Im Titel des Artikels, der im Schwäbischen Tagblatt über die Ergreifung des Täters berichtet, findet sich kein Verweis darauf, dass es sich um einen Asylbewerber handelt, erst im Fließtext wird der Täter als „Asylbewerber aus Gambia mit Wohnsitz in Tübingen" beschrieben (vgl. Schwäbisches Tagblatt 7.7.2017). Die Zeitung bietet vielfältigen Perspektiven auf das Ereignis Raum und weist pauschalisierende Aussagen über „Flüchtlinge“ oder „Gambier“ zurück. Boris Palmer wird mit der Äußerung zitiert, dass „Tübingen [...] eine der Städte [war], in denen Frauen sich ohne Angst frei bewegen konnten. Das hat sich leider verändert.“(ebd.). In einem „FaktenCheck“ widerspricht das Schwäbische Tagblatt dieser Aussage. Sie zitiert dazu den Leiter der Pressestelle des Polizeipräsidiums, der auf die Polizeiliche Kriminalstatistik verweist: „Die Tübinger Zahlen bei den Sexualdelikten sind in keiner Richtung auffällig oder gar alarmierend oder begründen gar eine erhöhte Gefährdungslage." Vielmehr lägen die Zahlen bei den Sexualdelikten in den Jahren 2015 und 2016 in der Stadt Tübingen bei je 55 Straftaten. 2008 waren es 72 Fälle. Auch die Zahl der Vergewaltigungen habe mit 12 Fällen im Vergleich zum Vorjahr nicht zugenommen (vgl. Schwäbisches Tagblatt 20.7.2017). Der Vorsitzende des Deutsch-Gambischen Kulturvereins in Tübingen kritisiert generalisierende Aussagen über Gambier in einem Leserbrief: „Doch auch, wenn einzelne Menschen aus Gambia hier eine Straftat begehen, sollten nicht alle Gambier [...] als Straftäter verdächtigt werden“ (Schwäbisches Tagblatt 14.7.2017). Die Zeitung zitiert darüber hinaus eine „schwarze Tübingerin“: „Die Aussage, Schwarze sind potenzielle Täter - das ist eine Form von Kriminalisierung von allen schwarzen Männern, die in Tübingen leben“ (Schwäbisches Tagblatt 20.7.2017).

Nach einer weiteren Vergewaltigung durch einen „dunkelhäutigen Mann“ im Alten Botanischen Garten berichtet das Schwäbische Tagblatt erneut über ein verändertes Sicherheitsgefühl in der Stadt. Unter der Überschrift „Die verunsicherte Stadt“ zitiert die Zeitung wiederum eine Aussage Boris Palmers („Viele Mädchen und Frauen fühlen sich in Tübingen nicht mehr 
sicher") und stellt dieser einen einordnenden Kommentar gegenüber, der sich auf Aussagen der Polizei bezieht („Bislang habe es in der ersten Jahreshälfte einen leichten Anstieg an angezeigten sexuellen Übergriffen gegenüber dem Vorjahr gegeben, insgesamt rechne man derzeit aber nicht mit einer großen Abweichung“). Darüber hinaus wird über Präventionsarbeit gegen sexuelle Übergriffe mit Mädchen und Jugendlichen berichtet, unter anderem über ein Präventionsprogramm in Schulen. Auslöser des Programms war ein Übergriff auf eine Elfjährige („Der Täter war übrigens kein Flüchtling, sondern Deutscher“, vgl. Schwäbisches Tagblatt 4.10.2017). Abschließend zitiert die Zeitung Aussagen der Bundesgeschäftsführerin von Terre des Femmes:

„Viele der Männer kommen aus patriarchalen Kulturen [...], wo sie nicht gerade häufig mit selbstbewussten Frauen konfrontiert werden. [...] Wir müssen diese kulturellen Unterschiede verstehen, erklären und dann Konsequenzen ziehen.“ (Schwäbisches Tagblatt 4.10.2017)[7]

Die Polizei verändert aufgrund des veränderten Sicherheitsgefühls im Alten Botanischen Garten, der Platanenallee oder dem Anlagenpark ihr Einsatzkonzept und zeigt verstärkte Präsenz. In Absprache mit der Staatsanwaltschaft sollen Täter in beschleunigten Verfahren zügig bestraft werden. Das habe auch einen erzieherischen Effekt. Angstbesetzte Räume sollten so ihren Schrecken verlieren (vgl. Schwäbisches Tagblatt 18.11.2017). In der Debatte um Flucht und Kriminalität hebt der Polizeipräsident soziale Ursachen hervor:

„Wir stellen da einen Unterschied fest zwischen jenen, die spüren, dass sie hier ankommen können, und denen, für die es nur eine Frage der Zeit ist, bis sie das Land verlassen müssen. [...] Und manche beschließen dann, eben noch mitzunehmen was geht - im wahrsten Sinne des Wortes, im Rewe, im Media-Markt.“ (Schwäbisches Tagblatt 18.11.2017)

Die Zahl der Sexualdelikte, berichtet das Schwäbische Tagblatt, steigt im Jahr 2017 auf 273 (2016: 211). Ähnlich hoch war sie in den vorangegangenen zehn Jahren nur 2013 (251). Darunter fallen Verfahren wegen sexueller Nötigung und Vergewaltigung (2017: 106 / 2016: 94). Zu berücksichtigen sei dabei, so das Schwäbische Tagblatt, dass der Bundestag das Sexualstrafrecht 2016 verschärfte. Körperliche Berührung mit sexuellem Bezug (,Grapschen') gelte seither als sexuelle Belästigung. Der Straftatbestand der Vergewaltigung sei auf Taten ausgeweitet worden, bei denen sich das Opfer nicht körperlich wehrt, sondern nur verbal (vgl. Schwäbisches Tagblatt 23.3.2018).

Die Berichterstattung des Schwäbischen Tagblatts weist übergreifend kaum Kennzeichen einer Moralpanik auf. Sie ist durch eine differenzierende Perspektive geprägt, die pauschalisierende Aussagen kontextualisiert (z. B. über den Verweis auf polizeiliche Statistiken). Darüber hinaus wird über Folgen von Alltagsrassismus für dessen Opfer berichtet (z. B. „Kriminalisierung von allen schwarzen Männern“). Eine Ethnisierung von Sexismus wird vermieden (z. B. „Täter [...] kein Flüchtling, sondern Deutscher“). Möglichen ,kulturellen' Erklärungsfaktoren für sexualisierte Gewalt wird Raum gegeben (z. B. „Männer kommen aus patriarchalen Kulturen“), ohne dass sie die Debatte dominieren. Als Expert_innen werden 
Frauenrechtsorganisationen, Präventionsprojekte und Beratungsstellen zitiert, wodurch die Opferperspektive in der Berichterstattung eine wichtige Rolle spielt.

Boris Palmer kommentiert das Ereignis auf Facebook wie folgt[8]:

„Vergewaltiger aus Gambia gefasst. Wir brauchen auch bei der Tübinger Polizei mehr Stellen. Tübingen war einer der Städte, in denen Frauen sich ohne Angst frei bewegen konnten. Das hat sich leider verändert. [...] Ist es wichtig, dass der Mann ein Asylbewerber und ein junger Mann war? Ja. Die Anzahl vergleichbarer Fälle ist einfach zu hoch, um das als völlig normal abzutun. Die Lebensumstände junger Männer ohne jede soziale Anbindung die in unserem Land untätig auf Klarheit über ihre Zukunft warten, machen solche Reaktionen wahrscheinlich. Davor zu warnen vor zwei Jahren fast ein Sakrileg. Es wird Zeit, diese Probleme zu lösen“.

Der Beitrag erhält 746 Reaktionen und wird 145-mal geteilt. Von 409 Kommentaren wurden 236 von männlichen Profilen verfasst (57,70 Prozent), davon 34 von Boris Palmer (8,31 Prozent). 162 wurden von weiblichen Profilen verfasst (39,61 Prozent) und 11 von Profilen, deren Namen nicht klar zugeordnet werden konnten (Stand: 13.3.2018).

Die im Beitrag selbst angelegte Deutung, dass der Status als Asylbewerber entscheidend für das Problem der Vergewaltigungen sei, wird in der Debatte vielfach aufgegriffen. Flüchtlings- und migrationspolitische Debatten dominieren den Diskurs. Das Asylrecht wird dabei wiederholt mit einem „Gast- und Aufenthaltsrecht“ (Manfred S.) gleichgesetzt. Geflüchteten wird die Schutzbedürftigkeit teils generell abgesprochen (z. B. „TaschegeldFlüchtlinge"; Meira E.). Asylbewerber aus Gambia werden als spezifisches Problem konstruiert (z. B. „Gambier sind extrem auffällig. Jeder weiß das.“; Ron J.). Als Konsequenz werden Forderungen nach einer raschen Abschiebung (krimineller und/oder aller) Geflüchteter geäußert: „Sofort abschieben!“ (Harpprecht V.).

Die Diskussion über Vergewaltigungen ist teilweise von einer (in zahlreichen Fällen rassistischen und entmenschlichenden) Ethnisierung von Sexismus gekennzeichnet (z. B. „Das sind alles junge Afrikaner, Moslems, also fast alles Männer, man spart sich das Geld für das Bordell und vergewaltigt Deutsche Frauen.“; Frank S. oder „genau,immer ist der westliche Einfluss schuld an irgendwelchen primitiven ,Annäherungsversuchen' der Männer aus Gambia...Dass die selbigen den ganzen Tag wie die wilden Karnickel Frauen ,begatten ' ist dir in deiner linksüberschwemmten Dachrinne wohl abhanden gekommen....."; Magdalene H.). Die hier sichtbar werdende Figur des entmenschlichten und hypersexualisierten ,Afrikaners' weist eine deutliche Kontinuität zu kolonial-rassistischen Stereotypen auf (vgl. Hall 2004). Im Zuge der durch rassistische Bilder von ,Muslimen“ oder ,Afrikanern“ geprägten Ethnisierung von Sexismus und der Konstruktion entsprechender Fremdgruppen werden sexistische Macht- und Herrschaftsverhältnisse innerhalb der eigenen Gruppe unsichtbar gemacht. In Deutschland seien „[p]atriarchalische Anmaßungen“ bei jüngeren Männern „Ausnahme, nicht die Regel“ (Johannes S.). Es sei vor diesem Hintergrund eine „Unterstellung deutsche Männer wären genauso“ (Meira E.). 
Einzelne Beiträge verweisen demgegenüber kritisch auf die Allgegenwärtigkeit sexualisierter Gewalt, die durch eine Ethnisierung aus dem Blick gerate:

„Erfahrungsgemäß melden mehr als 3/4 aller Vergewaltigungsopfer die Straftat nicht! Daher hilft da auch keine Polizei! [...] Und ein weitaus größeres Problem, das Boris Palmer ausser Acht lässt, die meisten Vergewaltigungen finden innerhalb der eigenen Familie statt und das werden keine Abschiebungen und sonst welche Maßnahmen verhindern.“ (Svetlana K.)

Boris Palmer reagiert auf diese Kritik mit dem Vorwurf, diese „Art der Relativierung“ sei „blind für die reale Entwicklung und verhöhnt die Opfer“ (Boris Palmer). Während Männer in ihren Kommentaren häufiger zu einer Ethnisierung von Sexismus neigen und sich in Einzelfällen dezidiert antifeministisch äußern (z. B. „Femenfront“; Olav K.), wird in den Kommentaren von Frauen häufiger Kritik daran geäußert (z. B. „Es ist schon höchst interssant, wie plötzlich selbst die härtesten Machos zu Frauenrechtlern werden, wenn es um Ausländer geht.“; Agnes R.).

Der Nexus zwischen Vergewaltigungen und Asylbewerbern basiert auf einer Mischung aus selektivem Bezug auf und der Auslegung von Statistiken (insbesondere der Kriminalstatistik des BKA) und anekdotischer Empirie. Der Bezug auf Statistiken ist dabei primär einer gefühlten bzw. „erfahrenen“ Wahrheit verpflichtet. Da die eigene Erfahrung über rationale Argumente gestellt wird, können unliebsame statistische Einwände jederzeit durch konkrete Beispiele und gefühlte Kausalbeziehungen, widerlegt 'werden, die als so offensichtlich wahr erfahren werden, dass sie keiner weiteren Legitimation bedürfen (und jeglicher Kritik standhalten):

„Natürlich kann man Auffälligkeiten in der PKS diskutieren. Aber man sollte dabei immer nach möglichen Ursachen fragen bzw. die Zahlen hinterfragen und nicht pauschal die statistische Auffälligkeit mit Straffälligkeit gleichsetzen. Doch genau das passiert in dem von Ihnen verlinkten Artikel und genau das macht auch Boris Palmer in seinem Post. Alles andere als seriös - leider!“ (Irene M.)

„Wieviel Beispiele, von Vergewaltigungen, Gewaltverbrechen, Ehrenmorden, Gewalt gegen Staatsdiener, geplante Terrooristische Anschläge, Einschüchterungen von braven Bürgern, Sozialbetrug etc etc,. etc. brauchst Du denn um zu begreifen, dass es einen direkten Zusammenhang gibt [...]?" (Werner F.)

„Wahrheit“ wird in der Folge in der Debatte mit der eigenen Meinung gleichgesetzt, sie könne durch einen ,gesunden Menschenverstand“ erspürt werden:

„Ihr müsst [...] Studien erstellen, ob es einen Zusammenhang zwischen Zuwanderung und wachsender Kriminalität ging. Da braucht man doch nicht kompliziert werden und Studien erstellen. Ein gesunder Menschverstand sieht den Zusammenhang auch so, aber er muss es wollen.“ (Werner F.)

Zur weiteren Begründung reicht gegebenenfalls bereits der abstrakte Verweis auf „Statistik“: „Oh mann dort drin wird die polizeiliche Kriminalstatistik 
zitiert. [...] Solche Mensche wie sie, die die Wahrheit komplett ignorieren sind ein großes Problem“ (Benjamin Y.).

In der Debatte finden sich Diskursstrategien, die typischerweise von neonazistischen und völkisch-konservativen Akteuren in sozialen Medien genutzt werden (vgl. Amadeu Antonio Stiftung 2017, Generation D 2018). Kritik wird durch aggressive persönliche Angriffe oder willkürliche Gegenvorwürfe beantwortet (,was sagt dann eine Frau, wenn ein Schwarzafrikaner diese massiv bedrängt?“; Micha F./ „Sie sagt haargenau dasselbe wie zu einem bierbäuchigen rotgesichtigen glatzköpfigen Teutonen mit Bierfahne [...].“; Gabriele K. / „Gabriele K., Ihr Kommentar ist übrigens äußert rassistisch“; Britta G.). Der neurechte Blog „Tichys Einblick“ dient wiederholt als Referenzpunkt. Eine konstante Wiederholung von Beiträgen simuliert eine besondere Relevanz rassistischer Argumente. In einigen Beiträgen werden sogar Kommentare des politischen Gegners imitiert, um entsprechende Argumente zu delegitimieren. Die imitierten Beiträge zielen offensichtlich darauf, die Stimmung in der Debatte gezielt emotional aufzuladen und eskalierend zuzuspitzen.

Insgesamt entsteht dadurch in der Debatte um den Beitrag von Boris Palmer ein aggressives Diskussionsklima, kritische Stimmen werden einschüchtert:

„hier findest du überwiegende Rassisten und wer hier Boris Palmer widerspricht und dann noch eine Frau ist, muss mit einem Shitstorm rechnen. [...] Es gibt hier auch einige Bots und Fakeprofile, mit Diskussion geht hier gar nichts.“ (Agnes R.)

Als Legitimation der Einschüchterungsstrategie dient wiederum die Referenz der politischen Korrektheit und die damit einhergehende Konstruktion einer vermeintlichen Realitätsferne jeglicher Kritik (z. B. „,weltfremde Träumer und Spinner“; Claudia S. / „linsgünversiffte[...] -- Vergewaltiger Befürworter“; Ulla S.). Wer Einwände gegen die dominante Diskursposition („Das Problem sind Asylbewerber") äußert, wird als politischer Gegner eingeordnet und diskursiv aggressiv ausgegrenzt (z. B. „sinnfreies rosa Gutmensch-Gerede“; Magdalena H.) / „Das kommt davon wenn man in der eigenen Welt lebt und von der Realen abgeschottetet ist“; Alfred P.). Vergewaltigungen werden teils als gerechte Strafe für eine liberale Migrations- und Flüchtlingspolitik angesehen (z. B. „Ja Mädel, das ist der Preis für Offen-grün-liberal. Damit müsst ihr euch jetzt abfinden.“; Christophorus J.).

Auch in diesem Fall weist die Debatte um den Eintrag von Boris Palmer deutliche Züge einer Moralpanik auf: Erstens wird der konkrete Vergewaltigungsfall pauschalisierend mit einer Gruppe verknüpft (z. B. „Asylbewerber und junger Mann“). Die konstruierte Gruppe wird anschließend durch rassistische Kommentare abgewertet und einem Generalverdacht ausgesetzt. Asylbewerber werden als unmoralisch und außerhalb der normativen Ordnung stehend eingeordnet, wodurch ihnen eine faire Behandlung und rechtliche Gleichstellung abgesprochen werden kann (vgl. Cohen 1980: 87). Das Asylrecht wird vor diesem Hintergrund grundlegend in Frage gestellt. Zweitens wird retrospektiv eine Idylle konstruiert, die einem gesellschaftlichen Verfallsprozess ausgesetzt sei. Drittens wird das Problem von Vergewaltigungen ethnisiert, wobei teilweise explizit auf (kolonial-)rassistische Stereotype zurückgegriffen wird. Vor allem von Frauen 
geäußerte Einwände gegen diese Engführung der Problematik sexualisierter Gewalt werden rhetorisch delegitimiert. Begründet wird dies viertens mit einer ,erfahrenen Wirklichkeit', die anekdotische Empirie mit einer selektiven Rezeption statistischer Daten (insbesondere der Polizeilichen Kriminalstatistik) kombiniert. Die eigene Position wird dabei als diskursiv nicht verhandelbare, einzig legitime Position der ,Wahrheit‘ inszeniert. Um diese ,Wahrheit‘ zu bekräftigen, werden selektiv ,Fakten` ausgewählt, die die gefühlte emotionale Bedeutung des Ereignisses stützen (vgl. Cohen 1980: 154-155). Nach Stuart Hall et al. ist die in diesem Sinne anti-aufklärerische Mobilisierung eines als homogen, natürlich und eindeutig inszenierten ,gesunden Menschenverstands' zur Legitimierung mit der eigenen Meinung übereinstimmender konservativer Wertvorstellungen, aber auch rassistischer, sozialchauvinistischer und (hetero-)sexistischer Ideologien ein typisches Element von Moralpaniken (vgl. Hall et al. 2002: 150-152). Einwände erscheinen dadurch „nicht mehr als Kritik, sondern nur noch als Ketzerei, als moralische Verfehlung“ (Seeßlen 2016) (,weltfremde Träumer und Spinner"). Die Moralpanik wird dabei fünftens zumindest teilweise durchaus absichtsvoll produziert (z. B. durch simulierte Debatten mit imitierten Kommentaren). Rassismus und eine aggressive Rhetorik gegenüber politischen Gegnern werden sechstens in der Debatte kaum in Frage gestellt, wodurch sie als eine legitime Meinung unter vielen erscheinen. Versuche, die Debatte über sachliche Argumente zu deeskalieren (z. B. „man sollte dabei immer nach möglichen Ursachen fragen bzw. die Zahlen hinterfragen“, „Sprache [...] verwenden, die keine ideologische Munition für die neue Rechte liefert“), bleiben vor diesem Hintergrund siebtens weitgehend erfolglos.

\section{Die Facebook-Seite Boris Palmers als Katalysator von Moralpaniken}

Die Debatten um Geflüchtete im Regierungsbezirk Tübingen, so zeigen die beiden in den vorherigen Abschnitten rekonstruierten Beispiele, sind vor allem auf der Facebook-Seite Boris Palmers durch stereotype und rassifizierende Fremdgruppenkonstruktionen geprägt. In der folgenden Tabelle (Tab. 1) werden die Diskurselemente in der Lokalpresse und auf der Facebook-Seite Boris Palmers für die zwei Ereignisse gegenübergestellt.

Das Konzept der Moralpanik bietet - so zeigen diese Ergebnisse durch seinen hegemonieanalytischen Fokus auf Sprechakte und Akteure eine produktive Erweiterung der wissenschaftlichen Debatte über Diskurse um Flucht und Migration in Deutschland. Es erlaubt, typische Topoi und Narrative des flüchtlingspolitischen Diskurses (z. B. Gewalt-, Kriminalitätsund Terrorängste oder kulturelle Fremdheit), autoritär-populistische und/ oder neonazistische Teilöffentlichkeiten sowie grundlegendere gesellschaftliche rassistische Stereotype und Deutungsmuster (z. B. des gewalttätigen und sexistischen muslimischen Flüchtlings) systematisch in der alltäglichen Praxis in den Blick zu nehmen. Gleichzeitig lenkt der Fokus auf Moralpaniken den Blick auf die vielschichtige Struktur des Diskurses, in der sich einerseits Momente der Konstruktion bestimmter Gruppen, ihrer rassistischen Abwertung und der Immunisierung gegen Kritik zu einem relativ kohärenten Diskurs verdichten, andererseits jedoch entsprechende Äußerungen auch in 


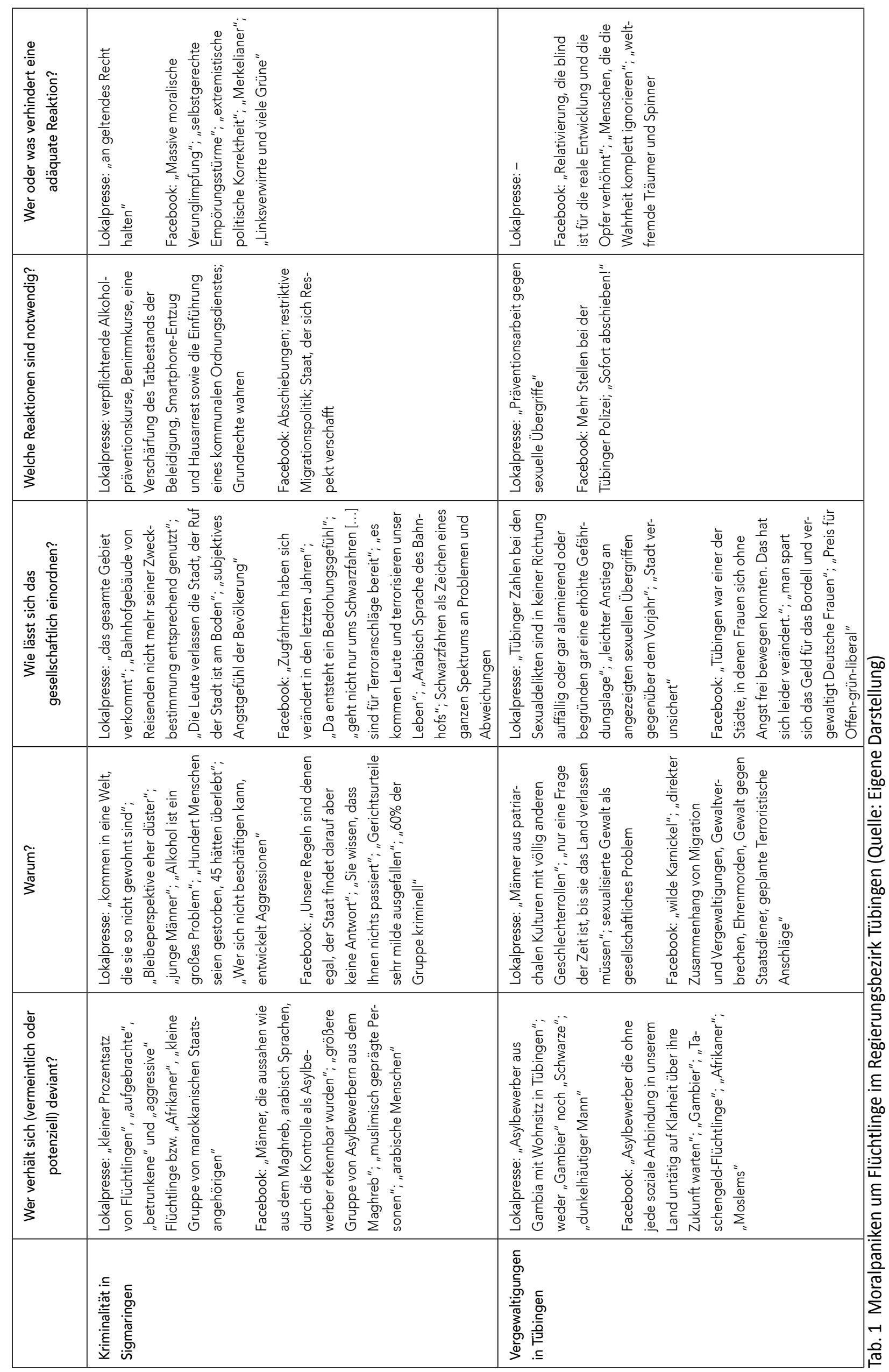


Frage gestellt und herausgefordert werden. Der Vergleich der Kommentare auf der Facebook-Seite mit Printmedien zeigt, dass diese eine je spezifische diskursive Selektivität bzw. ein jeweils eigenes Sagbarkeitsfeld aufweisen. Dominante Diskursposition in den Kommentaren auf der Facebook-Seite Boris Palmers ist eine diffamierende bis rassistische Perspektive gegenüber Geflüchteten und Migrant_innen. Die Seite wirkt dadurch als Katalysator von Moralpaniken. In Bezug auf Sprechpositionen bestätigen die Ergebnisse weitgehend die Befunde vorangegangener Analysen. Geflüchtete selbst kommen in der Berichterstattung der Printmedien nur am Rande zu Wort, die Debatten auf der Seite Boris Palmers verlaufen fast vollständig ohne eine Beteiligung von Geflüchteten.

Während der verwendete analytische Zugriff auf Moralpaniken sich als produktiv erweist, um flüchtlingspolitische Debatten auf synchroner Ebene differenziert in den Blick zu nehmen, ist er nur begrenzt in der Lage, die beschriebenen Diskurselemente diachron in Konjunkturen des Rassismus in Deutschland zu verorten. Für die implizit im Titel der „neuen Angst vorm Schwarzen Mann" angelegte Frage nach dem Wandel diskursiver Formationen wäre eine stärkere (rassismuskritische) Historisierung notwendig, als sie im Rahmen dieses Artikels geleistet werden konnte. Um die Auswirkungen der Moralpaniken und der radikalisierten Teilöffentlichkeit auf Facebook auf den städtischen Alltag in Blick zu nehmen, wäre weitere Forschung wünschenswert, die die Ergebnisse zu Erfahrungen derjenigen in Beziehung setzt, die im Zuge der Moralpaniken ausgegrenzt, rassistisch abgewertet und bedroht werden.

\section{Endnoten}

[1] Für wertvolle Hinweise zu einer vorherigen Version des Artikels danke ich zwei anonymen Gutachter_innen und Sarah Sott.

[2] Die Studie von Haller zeichnet sich durch ihr quantitatives empirisches Material zum Flüchtlingsdiskurs aus, die in der Studie vorgenommene Auswertung des Materials wurde hingegen zu Recht als tendenziös und suggestiv kritisiert (vgl. Horz 2017).

[3] Tatverdächtige sind dabei nicht mit Tätern gleichzusetzen, zum Beispiel da sich das Anzeigeverhalten gegenüber Verdächtigen unter anderem aufgrund rassistischer Deutungsmuster deutlich unterscheidet.

[4] Alle folgenden Zitate, sofern nicht anders markiert, sind dem Post und seiner Debatte entnommen (vgl. Palmer 5·5.2017).

[5] Rechtsschreib- und Grammatikfehler in den Facebook-Kommentaren werden im Artikel nicht mit [sic!] gekennzeichnet und sind jeweils aus dem Original übernommen.

[6] Zur Kritik der Objektivität entsprechender Statistiken vgl. Hall et al. (2002: 9-10).

[7] Diese Äußerung kann (z. B. in ihrer Rezeption durch Leser_innen) als Element einer (rassistischen) Moralpanik fungieren, da sie zumindest offen für eine Deutung von Kultur im Sinne einer naturalisierenden Kategorie ist und gleichzeitig in verallgemeinernder Form eine relativ homogene (Fremd-)Gruppe konstruiert („Viele der Männer“). Durch die Rassifizierung von Kultur in ethnopluralistischen Deutungsmustern, die weit über die Neue Rechte hinaus verbreitet sind, ist eine kulturelle Interpretation von Konflikten in gesellschaftspolitischen Auseinandersetzungen - insbesondere im Zuge von Moralpaniken (auch wenn diese auf Teilöffentlichkeiten beschränkt bleiben) - grundsätzlich mit der Gefahr verbunden, rassistische Ausgrenzung zu befördern oder zu legitimieren. Gleichzeitig können Kultur, Religion oder regionale Herkunft durchaus eine relevante Rolle für alltägliche Handlungsmuster (und deren wissenschaftliche Erklärung) spielen. Entscheidend 
ist mit Paula-Irene Villa ein nicht-deterministisches Kulturverständnis, dass Menschen als ,intersektional und komplex“ versteht (vgl. Hark/Villa 2017).

[8] Alle folgenden Zitate sind dem Post und seiner Debatte entnommen (vgl. Palmer 6.7.2017).

\section{Autor_innen}

Politikwissenschaftler, arbeitet unter anderem zu Geflüchteten auf dem Arbeitsmarkt, Willkommenskultur, radikaler Demokratie und autoritärem Populismus.

nikolai.huke@uni-tuebingen.de

\section{Literatur}

Amadeu Antonio Stiftung (2017): Toxische Narrative. https://www.amadeu-antoniostiftung.de/w/files/publikationen/monitoring-2017.pdf (letzter Zugriff am 30.1.2018).

Arendt, Florian / Brosius, Hans-Bernd / Hauck, Patricia (2017): Die Auswirkung des Schlüsselereignisses „Silvesternacht in Köln“ auf die Kriminalitätsberichterstattung. In: Publizistik 62/2, 135-152.

Butler, Judith (2011): Bodies in Alliance and the Politics of the Street. http://eipcp.net/ transversal/1011/butler/en/print (letzter Zugriff am 4.4.2012).

Cohen, Stanley (1980): Folk devils and moral panics. Oxford: Martin Robertson.

Cohen, Stanley (2011): Whose side were we on? The undeclared politics of moral panic theory. In: Crime Media Culture 7/3, 237-243.

Dietze, Gabriele (2016): Das ,Ereignis Köln‘. In: femina politica 1/2016, 93-102.

Drüeke, Ricarda (2016): Die TV-Berichterstattung in ARD und ZDF über die Silvesternacht 2015/16 in Köln. https://www.gwi-boell.de/sites/default/files/web_161122_e-paper_ gwi_medienanalysekoeln_v10o.pdf (letzter Zugriff am 24.1.2018).

Dubiel, Helmut (1986): Das Gespenst des Populismus. In: Helmut Dubiel (Hg.), Populismus und Aufklärung. Frankfurt/Main: Suhrkamp, 33-50.

Fitzgerald, Ian / Smoczynski, Rafal (2015): Anti-polish migrant moral panic in the UK. A response. In: Czech Sociological Review 51/3, 380.

Garland, David (2008): On the concept of moral panic. In: Crime Media Culture 4/1, 9-30.

Generation D (2018): Handbuch für Medienguerillas. https://www.hogesatzbau.de/wpcontent/uploads/2018/o1/HANDBUCH-FÜR-MEDIENGUERILLAS.pdf (letzter Zugriff am 27.8.2018).

Glorius, Birgit / Schondelmayer, Anne-Christin / Dörfel, Robinson (2018): „Wandel durch Annäherung“? Gesellschaftliche Konflikte im Kontext der Flüchtlingsunterbringung im ländlichen Sachsen. In: Simon Goebel / Thomas Fischer / Friedrich Kießling / Angela Treiber (Hg.), FluchtMigration und gesellschaftliche Transformationsprozesse. Wiesbaden: Springer, 111-140.

Goebel, Simon (2018): Dozieren, intervenieren, kapitulieren? Wissenschaftler_innen in politischen Talkshows über Flucht. In: Simon Goebel / Thomas Fischer / Friedrich Kießling / Angela Treiber (Hg.), FluchtMigration und gesellschaftliche Transformationsprozesse. Wiesbaden: Springer, 191-216.

Hall, Stuart (2004): Das Spektakel der ,Anderen‘. In: Stuart Hall (Hg.), Ideologie, Identität, Repräsentation. Ausgewählte Schriften 4. Hamburg: Argument, 108-166.

Hall, Stuart / Critcher, Chas / Jefferson, Tony / Clarke, John / Roberts, Brian (2002): Policing the crisis. London: Macmillan.

Haller, Michael (2017): Die „Flüchtlingskrise“ in den Medien. https://www.otto-brennerstiftung.de/fileadmin/user_data/stiftung/Aktuelles/AH93/AH_93_Haller_Web.pdf (letzter Zugriff am 17.1.2018).

Hark, Sabine / Villa, Paula-Irene (2017): Toxic. Interview mit Sabine Hark und Paula Villa zur Sexismusdebatte nach ,Köln`. https://www.zfmedienwissenschaft.de/online/ blog/toxic-interview-mit-sabine-hark-und-paula-villa-zur-sexismusdebatte-nach-koeln (letzter Zugriff am 17.1.2019).

Homes, Seth M. / Castañeda, Heide (2016): Representing the "European refugee crisis" in Germany and beyond. In: American Ethnologist 43/1, 12-24. 
Horz, Christine (2017): Zu positive Berichterstattung? Die Studie des Kommunikationswissenschaftlers Michael Haller zur „Flüchtlingsberichterstattung“ in deutschen „Leitmedien“. In: Global Media Journal 7 (2), 1-12.

Huke, Nikolai (2018): Feindbild Identitätspolitik und konservativer Rollback. In: Politikum 4/4, 14-21.

Jäger, Margarete / Wamper, Regina (Hg.) (2017): Von der Willkommenskultur zur Notstandsstimmung. http://www.diss-duisburg.de/wp-content/uploads/2017/o2/ DISS-2017-Von-der-Willkommenskultur-zur-Notstandsstimmung.pdf (letzter Zugriff am 31.1.2018).

McRobbie, Angela / Thornton, Sarah L. (1995): Rethinking 'moral panic' for multi-mediated Social worlds. In: The British Journal of Sociology 46/4, 559-574.

Mühe, Marieluise (2017): Rassistische Diskurse im Einwanderungsland Deutschland. http://www.polsoz.fu-berlin.de/polwiss/forschung/international/vorderer-orient/publikation/WP_serie/WP15_Marieluise_05.pdf (letzter Zugriff am 31.1.2018).

Perthus, Sophie / Belina, Bernd (2017): Policing the Crisis in Bautzen. In: Soziale Probleme 28/2, 241-259.

Salzborn, Samuel (2017): Angriff der Antidemokraten. Weinheim: Beltz Juventa.

Seeßlen, Georg (2016): Gefühlte Wirklichkeiten. In: Jungle World, 18.2.2016.

Tsianos, Vassilis (2013): Urbane Paniken. Zur Entstehung des antimuslimischen Urbanismus. In: Duygu Gürsel / Zülfukar Çetin / Allmende e.V. (Hg.), Wer MACHT Demo_kratie? Kritische Beiträge zu Migration und Machtverhältnissen. Münster: Ed. Assemblage, 23-43.

\section{Quellenverzeichnis}

Palmer, Boris 5.5.2017: Sigmaringen. Bahnhof. https://www.facebook.com/ob.boris. palmer/posts/1502458899793637 (letzter Zugriff am 13.3.2018).

Palmer, Boris 6.5.2017: Was bringen wir Asylbewerbern bei? https://www.facebook.com/ ob.boris.palmer/posts/1503209203051940 (letzter Zugriff am 13.3.2018).

Palmer, Boris 6.7.2017: Vergewaltiger aus Gambia gefasst. https://www.facebook.com/ ob.boris.palmer/posts/1571759436196916 (letzter Zugriff am 13.3.2018).

Schwäbische Zeitung 17.1.2017: LEA will Flüchtlinge nach Auseinandersetzung trennen. In: Schwäbische Zeitung vom 17.1.2017. URL: https://www.schwaebische.de/landkreis/ landkreis-sigmaringen/sigmaringen_artikel,-lea-will-flüchtlinge-nach-auseinandersetzung-trennen-_arid,10598203.html (letzter Zugriff am 02.05.2019).

Schwäbische Zeitung 19.1.2017: Djamil: „Es gibt in der Kaserne praktisch keine Privatsphäre“. In: Schwäbische Zeitung vom 19.1.2017. URL: https://www.schwaebische.de/ landkreis/landkreis-sigmaringen/sigmaringen_artikel,-djamil-es-gibt-in-der-kasernepraktisch-keine-privatsphäre-_arid,10599877.html (letzter Zugriff am 02.05.2019).

Schwäbische Zeitung 29.1.2017: Streit ruft Polizei-Spezialkräfte auf den Plan. In: Schwäbische Zeitung vom 29.01.2017. URL: https://www.schwaebische.de/landkreis/ landkreis-sigmaringen/sigmaringen_artikel,-streit-ruft-polizei-spezialkräfte-auf-denplan-_arid,10605261.html (letzter Zugriff am 02.05.2019).

Schwäbische Zeitung 10.2.2017: LEA: 30 Marokkaner machen Ärger. In. Schwäbische Zeitung vom 10.2.2017. URL: https://www.schwaebische.de/landkreis/landkreis-sigmaringen/sigmaringen_artikel,-lea-30-marokkaner-machen-ärger-_arid,10612547.html (letzter Zugriff am 02.05.2019).

Schwäbische Zeitung 16.2.2017: Kleine Gruppe Grund für Probleme in Lea Sigmaringen. In: Schwäbische Zeitung vom 16.2.2017. URL: https://www.schwaebische.de/sueden/ baden-wuerttemberg_artikel,-kleine-gruppe-grund-für-probleme-in-lea-sigmaringen-_ arid,10615857.html (letzter Zugriff am 02.05.2019).

Schwäbische Zeitung 16.2.2017: Regierungspräsident befürwortet Polizeiposten in der LEA. In: Schwäbische Zeitung vom 16.2.2017. URL: https://www.schwaebische.de/landkreis/ landkreis-sigmaringen/sigmaringen_artikel,-regierungspräsident-befürwortet-polizeiposten-in-der-lea-_arid,10616325.html (letzter Zugriff am 02.05.2019).

Schwäbische Zeitung 17.2.2017: LEA: Drogen, Gewalt und Brandstiftung. In: Schwäbische Zeitung vom 17.2.2017. URL: https://www.schwaebische.de/landkreis/landkreis-sigmaringen/sigmaringen_artikel,-lea-drogen-gewalt-und-brandstiftung-_arid,10616425.html (letzter Zugriff am 02.05.2019). 
Schwäbische Zeitung 18.3.2017: Mehr Staftaten von Flüchtingen in Sigmaringen. In: Schwäbische Zeitung vom 18.3.2017. URL: https://www.schwaebische.de/landkreis/landkreissigmaringen/sigmaringen_artikel,-mehr-straftaten-von-flüchtlingen-in-sigmaringen-_ arid,10633647.html (letzter Zugriff am 02.05.2019).

Schwäbische Zeitung 23.3.2017: Jeder dritte Tatverdächtige ist ein Flüchtling. In: Schwäbische Zeitung vom 23.3.2017. URL: https://www.schwaebische.de/landkreis/landkreissigmaringen/sigmaringen_artikel,-jeder-dritte-tatverdächtige-ist-ein-flüchtling-_ arid,10636812.html (letzter Zugriff am 02.05.2019).

Schwäbische Zeitung 6.4.2017: Im Anwohnerrat begegnen sich Flüchtlinge und Bürger. In: Schwäbische Zeitung vom 6.4.2017. URL: https://www.schwaebische.de/landkreis/ landkreis-sigmaringen/sigmaringen_artikel,-im-anwohnerrat-begegnen-sich-flüchtlinge-und-bürger-_arid,10646038.html (letzter Zugriff am 02.05.2019).

Schwäbische Zeitung 3.7.2017: Polizei bestreitet Zunahme von Straftaten. In: Schwäbische Zeitung vom 3.7.2017. URL: https://www.schwaebische.de/landkreis/landkreissigmaringen/sigmaringen_artikel,-polizei-bestreitet-zunahme-von-straftaten-_ arid,10695049.html (letzter Zugriff am 02.05.2019).

Schwäbische Zeitung 20.11.2017: Die Jugendkriminalität im Landkreis Sigmaringen steigt. In: Schwäbische Zeitung vom 20.11.2017. URL: https://www.schwaebische.de/landkreis/ landkreis-sigmaringen/sigmaringen_artikel,-die-jugendkriminalität-im-landkreissigmaringen-steigt-_arid,10778459.html (letzter Zugriff am 02.05.2019).

Schwäbische Zeitung 29.11.2017: Bahnhof in Sigmaringen wird zum Brennpunkt. In: Schwäbische Zeitung vom 29.11.2017. URL: https://www.schwaebische.de/landkreis/ landkreis-sigmaringen/sigmaringen_artikel,-bahnhof-in-sigmaringen-wird-zum-brennpunkt-_arid,10777325.html (letzter Zugriff am 02.05.2019).

Schwäbische Zeitung 10.1.2018: Händler beschweren sich über Flüchtlinge. In: Schwäbische Zeitung vom 10.1.2018. URL: https://www.schwaebische.de/landkreis/landkreissigmaringen/sigmaringen_artikel,-händler-beschweren-sich-über-flüchtlinge-_ arid,10799292.html (letzter Zugriff am 02.05.2019).

Schwäbische Zeitung 8.2.2018: „Unerträglich“: Sigmaringen reagiert auf Kritik. In: Schwäbische Zeitung vom 8.2.2018. URL: https://www.schwaebische.de/landkreis/landkreissigmaringen/sigmaringen_artikel,-unerträglich-sigmaringen-reagiert-auf-kritik-_ arid,10815506.html (letzter Zugriff am 02.05.2019).

Schwäbische Zeitung 9.2.2018: Flüchtlinge: Bürgermeister fordert Durchgreifen. In: Schwäbische Zeitung vom 9.2.2018. URL: https://www.schwaebische.de/landkreis/landkreissigmaringen/sigmaringen_artikel,-flüchtlinge-bürgermeister-fordert-durchgreifen-_ arid,10816577.html (letzter Zugriff am 02.05.2019).

Schwäbische Zeitung 21.2.2018: Sanktionen gegen Flüchtlinge: Rückendeckung für Bürgermeister. In: Schwäbische Zeitung vom 21.2.2018. URL: https://www.schwaebische.de/ landkreis/landkreis-sigmaringen/sigmaringen_artikel,-sanktionen-gegen-flüchtlingerückendeckung-für-bürgermeister-_arid,10822787.html (letzter Zugriff am 02.05.2019).

Schwäbische Zeitung 4.3.2018: Strobl gegen Hausarrest für auffällige Flüchtlinge. In: Schwäbische Zeitung vom 4.3.2018. URL: https://www.schwaebische.de/landkreis/ landkreis-sigmaringen/sigmaringen_artikel,-strobl-gegen-hausarrest-für-auffälligeflüchtlinge-_arid,10829257.html (letzter Zugriff am 02.05.2019).

Schwäbische Zeitung 29.3.2018: Flüchtlingskriminalität am Bahnhof Sigmaringen: Die Stimmung ist angespannt. In: Schwäbische Zeitung vom 29.3.2018. URL: https://www. schwaebische.de/landkreis/landkreis-sigmaringen/sigmaringen_artikel,-flüchtlingskriminalität-am-bahnhof-sigmaringen-die-stimmung-ist-angespannt-_arid,10843541.html (letzter Zugriff am 02.05.2019).

Schwäbische Zeitung 19.4.2018: Polizeichef: „Sigmaringen ist eine sichere Stadt“. In: Schwäbische Zeitung vom 19.4.2018. URL: https://www.schwaebische.de/landkreis/ landkreis-sigmaringen/sigmaringen_artikel,-polizeichef-sigmaringen-ist-eine-sicherestadt-_arid,10855392.html (letzter Zugriff am 02.05.2019).

Schwäbische Zeitung 16.5.2018: Flüchtlingskriminalität in Sigmaringen nimmt ab. In: Schwäbische Zeitung vom 16.5.2018. URL: https://www.schwaebische.de/landkreis/ landkreis-sigmaringen/sigmaringen_artikel,-flüchtlingskriminalität-in-sigmaringennimmt-ab-_arid,10870831.html (letzter Zugriff am 02.05.2019).

Schwäbisches Tagblatt 7.7.2017: Vergewaltigungen aufgeklärt. In: Schwäbisches Tagblatt vom 7.7.2017.

Schwäbisches Tagblatt 14.7.2017: Zur Verurteilung aller. In: Schwäbisches Tagblatt vom 14.7.2017. 
Schwäbisches Tagblatt 20.7.2017: Wirklich wahr, Herr Palmer?. In: Schwäbisches Tagblatt vom 20.7.2017. URL: https://www.tagblatt.de/Nachrichten/Rassismus-Vorwuerfegegen-Tuebingens-OB-Boris-Palmer- $t 54 . h t m l$ (letzter Zugriff am 02.05.2019).

Schwäbisches Tagblatt 4.10.2017: Die verunsicherte Stadt. In: Schwäbisches Tagblatt vom 4.10.2017. URL: https://www.tagblatt.de/Nachrichten/Die-verunsicherte-Stadt-348559. html (letzter Zugriff am 02.05.2019).

Schwäbisches Tagblatt 18.11.2017: Das Sicherheitsgefühl der Menschen ist ihm heilig. In: Schwäbisches Tagblatt vom 18.11.2017. URL: https://www.tagblatt.de/Nachrichten/ Das-Sicherheitsgefuehl-der-Menschen-ist-ihm-heilig-353910.html (letzter Zugriff am 02.05.2019).

Schwäbisches Tagblatt 23.3.2018: Gewaltdelikte sind auf Zehnjahreshoch. In: Schwäbisches Tagblatt vom 23.3.2018. URL: https://www.tagblatt.de/Nachrichten/Die-TuebingerStaatsanwaltschaft-legte-ihre-Jahresbilanz-2017-vor-367933.html (letzter Zugriff am 02.05.2019).

\section{„The new fear of the black man“. Moral panics as a reaction to refugees in the district of Tübingen, Germany}

The concept of moral panic describes a dynamic in which a specific group is constructed as homogenous, alien, deviant and danger to the moral order of society. The article presents two case studies of moral panics surrounding the arrival of refugees in Germany: 1) Public debates around crime and the refugee shelter in Sigmaringen and 2) discussions about rape and refugees in Tübingen. Typical elements of the moral panics in both cases are long chains of equivalence, a generalized suspicion against targeted groups, the retrospective construction of an endangered idyll, the reference to a supposed common sense in opposition to a naive political correctness as well as the claim of permissive and helpless legal and police authorities. Social media such as Facebookfacilitates a quick diffusion of moral panics. Strategic interventions of openly racist accounts play an important role in this dynamic. 